\title{
Partial Paschen-Back splitting of Si II and Si III lines in magnetic CP stars $\dagger$
}

\author{
Viktor Khalack ${ }^{1}$ and John Landstreet ${ }^{2,3}$ \\ ${ }^{1}$ Université de Moncton, Moncton, N.-B., Canada, email: khalakv@umoncton.ca \\ ${ }^{2}$ University of Western Ontario, London, Canada \\ ${ }^{3}$ Armagh Observatory, Armagh, Northern Ireland - United Kingdom
}

\begin{abstract}
A number of prominent spectral lines in the spectra of magnetic A and B main sequence stars are produced by closely spaced doublets or triplets. Depending on the strength and orientation of magnetic field, the PPB magnetic splitting can result in the Stokes $I$ profiles of a spectral line that differ significantly from those predicted by the theory of Zeeman effect. Such lines should be treated using the theory of the partial Paschen-Back (PPB) effect. To estimate the error introduced by the use of the Zeeman approximation, numerical simulations have been performed for Si II and Si III lines assuming an oblique rotator model. The analysis indicates that for high precision studies of some spectral lines the PPB approach should be used if the field strength at the magnetic poles is $B_{\mathrm{p}}>6-10 \mathrm{kG}$ and $V \sin i<15 \mathrm{~km} \mathrm{~s}^{-1}$. In the case of the Si II line $5041 \AA$, the difference between the simulated PPB and Zeeman profiles is caused by a significant contribution from a so called "ghost" line. The Stokes $I$ and $V$ profiles of this particular line simulated in the PPB regime provide a significantly better fit to the observed profiles in the spectrum of the magnetic Ap star HD 318107 than the profiles calculated assuming the Zeeman effect.
\end{abstract}

Keywords. atomic processes - magnetic fields - line: profiles - stars: chemically peculiar stars: magnetic fields - stars: individual: HD318107

\section{Introduction}

In the analysis and modelling of spectral lines observed in magnetic upper main sequence stars, it is normally assumed that the splitting of the lines is correctly described by the anomalous Zeeman effect. However, in some cases the fine structure splitting of one or both levels involved in a transition is very small, and magnetic fields found in some stars are large enough to produce magnetic splitting comparable in size to this small fine structure splitting. In these cases, the splitting of the line should be calculated taking into account both the fine structure splitting and the magnetic splitting simultaneously. This regime is known as the incomplete or partial Paschen-Back (PPB) effect (Paschen $\&$ Back 1921). The partial Paschen-Back regime can occur when observed spectral line profiles are created by closely spaced doublets or triplets.

\section{Comparison of PPB and Zeeman profiles}

Calculation of the Paschen-Back splitting of spectral lines has been incorporated into the ZEEMAN2 code (Khalack \& Landstreet 2012), which allows us to simulate a line

$\dagger$ Based on observations obtained at the Canada-France-Hawaii Telescope (CFHT) which is operated by the National Research Council of Canada, the Institut National des Sciences de l'Univers of the Centre National de la Recherche Scientique of France, and the University of Hawaii. 

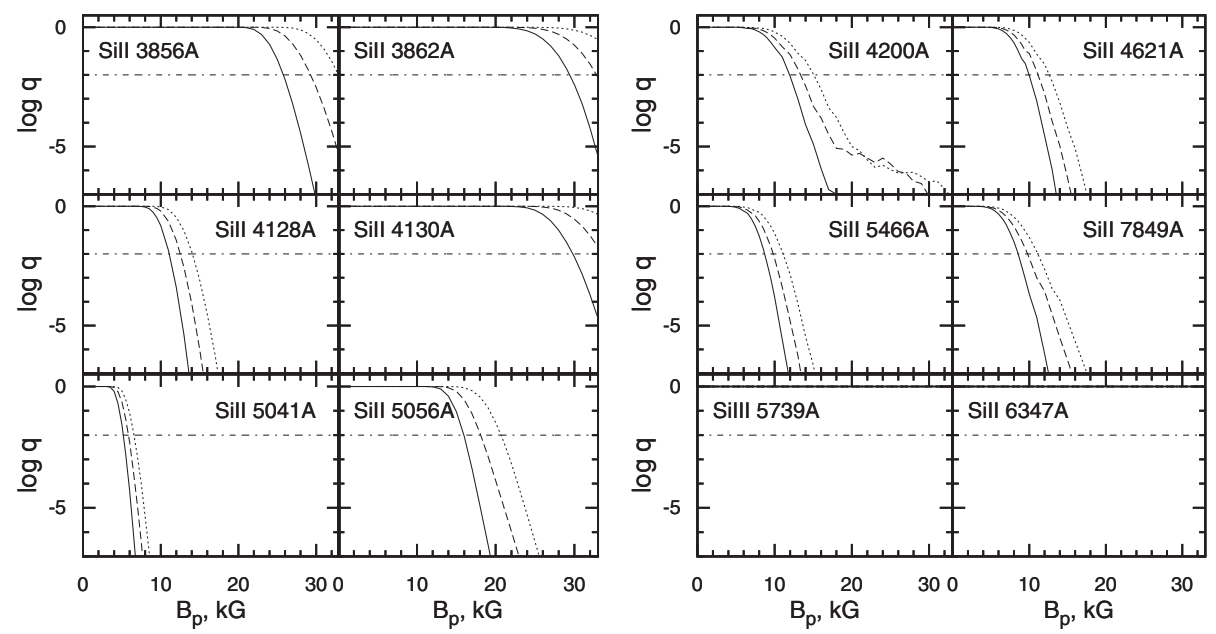

Figure 1. Logarithm of the probability that the noise with $\sigma=0.04(\mathrm{~S} / \mathrm{N}=250)$ is masking the difference between the Stokes $I$ calculated assuming PPB and Zeeman splitting, $\log \left[N_{\mathrm{Si}} / N_{\mathrm{H}}\right]=-3.5$ and $V \sin i=1 \mathrm{~km} \mathrm{~s}^{-1}$. The continuous, dashed, and dotted lines correspond to the cases where the axis of magnetic dipole forms an angle $\alpha=0^{\circ}, 45^{\circ}$, and $90^{\circ}$ with the line of sight, respectively. The horizontal dash-dotted line corresponds to the probability $q=0.01$. The bottom of the right-hand panel presents the examples of Si II and Si III lines that show $q \approx 1$ for Stokes $I$ profiles with little or no dependence on the field strength.

profile composed of several spectral lines (blends), some of which are split in the PaschenBack regime, while the others are split in the Zeeman regime. Initially, this code was created by Landstreet (1988) for the simulation of polarimetric (Stokes IVQU ) line profiles, and was later modified by Khalack \& Wade (2006), who added an automatic minimization of the model parameters using the downhill simplex method. The procedure for calculation of Paschen-Back splitting takes into account the magnetically perturbed energy levels and determines the respective air wavelength and oscillator strength of components, based on the term configurations and the total strength of all lines in the multiplet under consideration.

The simulation of Stokes $I$ profiles of Si II and Si III lines is carried for a star with $T_{\text {eff }}=13000 \mathrm{~K}, \log g=4.0$, zero microturbulent velocity, and an oblique rotator model with a dipolar magnetic field structure, assuming that the field strength at the magnetic pole is $B_{p}$ and the the axis of magnetic dipole forms an angle $\alpha=0^{\circ}, 45^{\circ}$ and $90^{\circ}$ with the line of sight. The observed Stokes $I$ spectra are usually contaminated by observational noise $\sigma \approx \frac{1}{S / N}$. To decide whether the difference between the simulated PPB and Zeeman profiles can be confidently detected above the given noise level we use the chi square probability function (Abramowitz \& Stegun 1972)

$$
q\left(\chi^{2} \mid \nu\right)=1-p\left(\chi^{2} \mid \nu\right)=\left[\Gamma\left(\frac{\nu}{2}\right)\right]^{-1} \int_{\chi^{2} / 2}^{\infty} t^{\frac{\nu}{2}-1} e^{-t} d t
$$

where $\nu$ is the number of resolved elements in the analyzed profile and

$$
\chi^{2}=\frac{1}{\nu \sigma^{2}} \sum_{i=1}^{\nu}\left(I_{i}^{P B}-I_{i}^{Z e}\right)^{2},
$$

where $I_{i}^{\mathrm{PB}}$ and $I_{i}^{\mathrm{Ze}}$ represent the intensity of the Stokes $I$ profiles at a wavelength point $i$ calculated with the assumption of the PPB and Zeeman splitting, respectively. For $\chi^{2}=35.7$ and $\nu=20$ the probability that PPB and Zeeman profiles are indistinguishable 

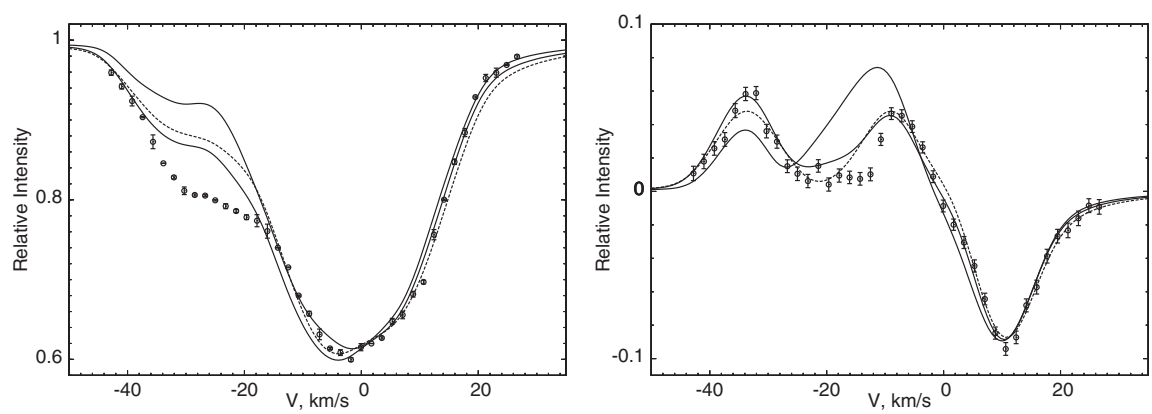

Figure 2. The best fit model for the Stokes $I$ (left) and Stokes $V$ (right) profiles of Si II $5041 \AA$ line in the Zeeman regime (dotted line) and PPB regime (continuous line) in combination with the FeI and Fe II blends. Open circles define the observed Stokes I and V profiles of Si II $5041 \AA$ line and the dashed curve provides the best fit results for this line assuming PPB splitting without Fe blends. The results for the Zeeman splitting of Si II $5041 \AA$ line without the contribution from the iron lines are not shown here.

Table 1. Approximation of Si II $5041 \AA$ line observed in the spectrum of HD 318107 (phase 0.991) employing the PPB and Zeeman effects (see text for details).

\begin{tabular}{lccccr}
\hline$\lambda, \AA$ & Splitting & $\log \left[N_{\mathrm{Si}} / N_{\mathrm{H}}\right]$ & $V \sin (i), \mathrm{km} \mathrm{s}^{-1}$ & $V_{r}, \mathrm{~km} \mathrm{~s}^{-1}$ & $\chi^{2} / \nu$ \\
\hline 5041 & $\mathrm{PPB}$ & $-3.57 \pm 0.15$ & $7.9 \pm 1.0$ & $-8.4 \pm 1.0$ & 16.5 \\
5041 & $\mathrm{PPB}+\mathrm{Fe}$ & $-3.80 \pm 0.15$ & $7.3 \pm 1.5$ & $-8.1 \pm 1.0$ & 9.8 \\
5041 & Zeeman & $-3.74 \pm 0.15$ & $7.5 \pm 0.8$ & $-8.0 \pm 1.0$ & 70.7 \\
5041 & Zeeman+Fe & $-3.94 \pm 0.15$ & $7.5 \pm 0.8$ & $-7.7 \pm 1.0$ & 40.4 \\
\hline
\end{tabular}

is $q<0.01$. This confidence level seems to be quite robust to perform an evaluation of the difference between the PPB and Zeeman profiles for individual lines (see Fig. 1).

The Si II $5957 \AA, 5979 \AA, 6347 \AA, 6371 \AA$ and Si III $4552 \AA, 4567 \AA, 4574 \AA$ lines provide $\log q$ close to zero with almost no dependence on the magnetic field strength for the Stokes $I$ profiles. For the Si II $3856 \AA, 3862 \AA$ and $4130 \AA$ lines there is no dependence of $\log q$ on magnetic field strength for $B_{\mathrm{p}}<20 \mathrm{kG}$. Meanwhile, for the Si II $4128 \AA, 4200 \AA, 4621 \AA$, $5041 \AA, 5466 \AA$ and $7849 \AA$ lines, the simulated PPB and Zeeman profiles appear to be different $\left(q<0.01\right.$ assuming $\mathrm{S} / \mathrm{N}=250$ and $\left.V \sin i=1 \mathrm{~km} \mathrm{~s}^{-1}\right)$ for a magnetic field strength $B_{\mathrm{p}}=5-15 \mathrm{kG}$.

We have also studied the dependence of the probability (see Eq. 2.1) on the magnetic field strength for higher rotational velocities $\left(1 \mathrm{~km} \mathrm{~s}^{-1}<V \sin i<30 \mathrm{~km} \mathrm{~s}^{-1}\right)$. The analysis of the Si II $4128 \AA, 5041 \AA, 5466 \AA$ and $7849 \AA$ lines with $\mathrm{S} / \mathrm{N}=250$ shows that the application of the PPB splitting for the simulation of Stokes $I$ profiles remains important in stars with $V \sin i<15 \mathrm{~km} \mathrm{~s}^{-1}$ when the field strength is $B_{\mathrm{p}}>10-15 \mathrm{kG}$.

From the bottom of the left-hand panel of Fig. 1 we can see that the PPB effect becomes important for simulations of the Stokes $I$ profile of Si II $5041 \AA$ line when $B_{p}>5$ $\mathrm{kG}$. For this line the difference between the simulated PPB and Zeeman profiles appears due to the so called "ghost" line with $|\Delta J|=2$ (Khalack \& Landstreet 2012). To test the obtained theoretical results, the Stokes I and V profiles of this particular line were analysed for the magnetic Ap star HD318107 $\left(T_{\text {eff }}=11800 \mathrm{~K}, \log g=4.2\right)$ using both Zeeman and PPB splitting. The geometry of the magnetic field model used for this star is described by the following parameters: $i=22^{\circ}, \beta=65^{\circ}, B_{\mathrm{d}}=25.6 \mathrm{kG}, B_{\mathrm{q}}=-12.8 \mathrm{kG}$, $B_{\mathrm{o}}=0.9 \mathrm{kG}$ that we have adopted from Bailey et al. (2011). The weak blending lines Fe I $5040.85 \AA, 5040.90 \AA$ and Fe II $5040.76 \AA$ are also taken into account during the 
simulation, assuming their Zeeman magnetic splitting. From Fig. 2 one can see that the best fit of the observed data is obtained assuming PPB splitting of Si II $5041 \AA$ line and the contribution of the iron blends (see second line in the Tabl. 1). The obtained data are close to the results derived by Bailey et al. (2011) from a complex analysis of different spectral lines for this star. The remaining differences between the PPB profiles and the observed spectra may be partially explained in terms of a more complicated actual magnetic field structure and/or horizontal and vertical stratification of the silicon abundance.

\section{Summary}

If the available polarimetric spectra of the stars with strong magnetic field have $\mathrm{S} / \mathrm{N}$ ratio higher than 250, the use of PPB splitting during the analysis of spectral lines is necessary to obtain precise results in the framework of an assumed model for the abundance map and the magnetic field structure. In particular, Stokes $I$ profiles of some Si II lines, when calculated with the PPB splitting, differ significantly from those calculated with the Zeeman effect (see Fig. 1). This difference appears due to the different relative intensities and positions of split $\sigma$ and $\pi$-components in the PPB and Zeeman regimes, and due to the "ghost" lines $(|\Delta J| \geqslant 2)$ as in the case of Si II $5041 \AA$ line (Khalack \& Landstreet 2012). For this line profile a contribution from the "ghost" lines becomes significant for $B_{\mathrm{p}}>5 \mathrm{kG}$ and an enhanced (by 1 dex) silicon abundance.

\section{References}

Abramowitz, M. \& Stegun, I. A. 1972, Handbook of Mathematical Functions, (10th ed. National Bureau of Standards, Washington), p. 940

Bailey, J. D., Landstreet, J. D., Bagnulo, S. et al. 2011, A\& A, 535, 25

Khalack, V. \& Landstreet, J. 2012, MNRAS, 427, 569

Khalack, V. \& Wade, G. 2006, A\&A, 450, 1157

Landstreet, J. D. 1988, ApJ, 326, 967

Paschen, F. \& Back, E. 1921, Physica, 1, 261 